\title{
A gestão da competitividade industrial por meio da aplicação dos métodos UP e multicritério no setor frigorífico de bovinos
}

\author{
The management of industrial competitiveness through the application \\ of methods UP and multi-criteria in a bovine slaughterhouse
}

$\begin{array}{llll}\text { R. Costa } & \text { J. Siluk } & \text { A. Neuenfeldt Júnior } & \text { M. Soliman }\end{array}$

Recibido 2 de agosto de 2013, aceptado 29 de septiembre de 2014

Received: August 2, 2013 Accepted: September 29, 2014

\begin{abstract}
RESUMO
O presente trabalho busca mostrar o desenvolvimento de uma modelagem capaz de revelar os custos dispendidos com a manufatura em uma empresa do ramo frigorífico, por meio da aplicação do método de Unidade de Produção em conjunto com conceitos a respeito dos métodos multicritérios. A fim de verificar o seu comportamento na prática, buscou-se aplicar o modelo em uma empresa situada no estado do Rio Grande do Sul, Brasil entre o período de Março a Agosto de 2012, visualizando-se, ao final, que o produto denominado como "Costilhar" é o mais oneroso $\left(C Q_{6_{b}}=\mathrm{R} \$ 1,05 \cong \mathrm{US} \$ 0,43\right)$, devido principalmente ao tempo necessário para o desenvolvimento dos seus processos $\left(\partial_{6_{b}}=280,7 \mathrm{UP}\right)$. Em específico, encontraram-sevalores satisfatórios em relação ao contexto de produção para quatro dos seis períodos avaliados, em relação ao valor estipulado como ideal $\left(C Q_{f b}=\mathrm{R} \$ 0,65\right)$, no qual demonstra a boa situação da empresa perante o contexto abordado.
\end{abstract}

Palavras-chaves: Gestão estratégica, competitividade, unidades de produção, custos de produção, métodos multicritério, frigorífico de bovinos.

\begin{abstract}
The dynamism of the modern world has forced companies to develop many tools and techniques to assist in the administration. In this sense, the present work aims to show the development of a model capable of revealing the costs expended to manufacture on a coil cold storage sector, through the application of the Unit of Production Method, in conjunction with concepts about multi-criteria methods. In order to verify the behavior in practice, we sought to apply the model in a company located in the state of Rio Grande do Sul, Brazil between the period from March to August 2012, visualizing in the end that "Sidecut" is the most expensive $\left(C Q_{6_{b}}=\mathrm{R} \$ 1,05 \cong \mathrm{US} \$ 0,43\right)$, mainly due to time required for the development of its processes $\left(\partial_{6_{b}}=280,7 \mathrm{UP}\right)$. Besides that, satisfactory values were found for four of the six periods evaluated in relation to the amount stipulated as ideal $\left(C Q_{f b}=\mathrm{R} \$ 0,65\right)$, which demonstrates the good business situation of the company in relation to the context.
\end{abstract}

Keywords: Strategic management, competitiveness, unit of production, production costs, multi-criteria methods, bovine cold store.

1 Universidade Federal de Santa Maria. Avenida Roraima, 1000. Centro de Tecnologia-sala 300. Código postal: 97105-900. Santa Maria, Brasil. E-mail: robsonperanconi@bol.com.br; jsiluk@ufsm.br; alvjr2002@hotmail.com; marlonsoliman@gmail.com

2 Universidade de Santa Cruz do Sul. Avenida Independência, 2293. Código postal: 96815-900. Santa Cruz do Sul, Brasil. E-mail: elpidio@unisc.br 


\section{INTRODUÇÃO}

Frente às dificuldades que o mundo globalizado apresenta e a velocidade em que essas se desenvolvem, pode-se observar que é imprescindível para o sucesso de uma organização o correto gerencimanento do controle de suas finanças, servindo de subsídio para o desenvolvimento da gestão e tomada de decisão, sendo possível afirmar que é na Unidade de Produção (UP) das empresas que são exercidas as atividades que dizem respeito à fabricação propriamente dita dos produtos, responsáveis diretamente pelo seu nível de sucesso ou fracasso em relação ao mercado [1-2].

Logo, para que ocorra uma administração eficiente destas, é necessário a utilização de métodos e ferramentas adequadas, visando a otimização dos resultados, fato este que se contrapõe no momento da verificação do gerencimanento dos custos de produção desenvolvido sob critérios tradicionais, o qual persiste em manter controles baseados unicamente na experiência adquirida pelas pessoas com passar dos anos [2-4].

Desta forma, a empresa não leva em consideração os pressupostos que podem ser obtidos através do conhecimento de sua estrutura financeira, mentalidade mutável desde que elas encararem o conceito de produzir como uma unidade de negócios, com metas a serem alcançadas sob um controle sistemático dos processos [5-6].

Portanto, pode-se afirmar que um dos pontos mais relevantes observados na gestão empresarial é o controle efetivo dos custos industriais, pois estes são parâmetro para definir o grau de desempenho do negócio, dando assim uma visão detalhada dos gastos para que estes se tornem uma ferramenta significativa da gestão estratégica. Para que isso aconteça, é importante a utilização de ferramentas e métodos de custeio que visam o saber a respeito do montante financeiro que está realmente impedindo a maximização dos lucros [7-11].

Visando abordar o tema sob um enfoque inovativo, é possível afirmar que o objetivo do estudo é o desenvolvimento de uma modelagem capaz de verificar o perfil dos custos na manufatura com base na aplicação dos métodos de Unidade de Produção e multicritério, utilizando como piloto o caso de uma frigorífica situada no estado do Rio Grande do Sul, no período de Março a Agosto de 2012.
A abordagem ao tema se justifica frente ao cenário onde o alto índice de competitividade e as oscilações, à influência do mercado externo e interno, fazem com que as organizações tenham a sua disposição informações concretas sobre os reais dispêndios financeiros de fabricação, para que o processo de gestão estratégica e tomada de decisões aconteçam de forma eficiente, ressaltando assim a relevância tanto acadêmica-científica quanto profissional aplicada desta pesquisa.

Através do levantamento bibliográfico referente as produções científicas publicadas em meios científicos por intermédio de artigos científicos, foi possível constatar que a presente aplicação possui um grau de originalidade, visto que não houve a constatação de que estudos semelhantes tenhas sido conduzidos a respeito do tema apenas da apresentação de uma síntese desta pesquisa em [12].

Para tal levantamente houve a necessidade da utilização de uma ampla base de informações, de forma que a fim de suprir esta demanda utilizaou-se o reposotório de dados disponibilizado pelo Portal de periódicos da CAPES®. Esta contém em seu escopo publicações indexadas nos mais renomados editoriais científicos mundiais, como Web of Knowledge ${ }^{\circledR}$, Scopus ${ }^{\circledR}$ e SciELO®, por meio das palavras-chaves "unit of production method", "unit of production", "slaughterhouse multicriteria", "slaughterhouse AHP" and "bovine slaughterhouse", por um período de tempo indeterminado.

Após a mineração dos dados obtidos foram detectados ao total quarenta artigos relacionados ao tema, de maneira que três estão vinculados a "unit of production", onde [13] esclarecem sobre a natureza do problema encontrado nas empresas multiproduto e oferecer uma solução através da abordagem UP.

Enquanto isso, [14] promoveram uma discussão a respeito dos constructos para a construção modelos de controle da produção baseados no método UP, a partir da coexistência de dois pontos de vistas: a precisão do modelo, o que implica a construção de modelos ideais, e a necessidade da geração do conhecimento global, que está associado às diferentes visões do processo. Por fim, [15] apresenta uma nova ferramenta de gestão aplicado na Romênia depois de 1990, demoninada por "Controlling", aplicada ao caso da mensuração de desempenho do esforço produtivo para uma empresa localizada naquele país. 
O restante de artigos, e trinta e sete, estão vinculados ao "bovine slaughterhouse", de maneira que nenhum remete ao escopo da gestão estratégica, competitividade ou gestão dos recursos financeiros na produção de carne bovina.

\section{MÉTODO DE UNIDADE DE PRODUÇÃO}

Conforme os pressupostos descritos por [3], é possivel afirmar que o método Unidade de Produção (UP) foi criado para mensurar de maneira padronizada e detalhada as diversas formas de fabricação de materiais, a fim de conhecer o esforço aplicado a cada uma delas, sejam automatizadas ou não [16].

Com isso, o método serve como parâmetro para diversos setores empresariais, pois estes estarão constantemente sendo abastecidos com dados concretos e de suma importância para as atividades de tomada de decisões estratégicas, possibilitando ao final a classificação das operações industriais de acordo com as que realmente agregam valor para o sistema $[4,7,17]$.

A fundamentação do modelo é concebida de acordo com a noção de esforço produtivo, sendo tal dispêndio relacionado ao funcionamento da máquina, tarefas de natureza humana, rentabilização e outras direta ou indiretamente aplicadas, considerando como total o somatório de cada uma, que resultará na medida do esforço despendido pela fábrica para produzir $[6,8,18]$.

Para tanto, para se aplicar o método deve-se, primeiramente, transformar a fábrica em um "modelo matemático", medido em uma única unidade de medida (UP), dividindo-se a mesma em "postos operativos", de modo a se mensurar o esforço necessário para que cada uma destas realize complenitude as suas atividades, o que demanda a necessidade de se considerar os seguintes custos elementares de produção: mão-de-obra direta e indireta, depreciação, manutenção, energia elétrica, encargos sociais e materiais de consumo $[8,14,18]$.

Em um segundo momento é realizado o mapeamento dos processos em que cada produto necessita ser submetido para a sua concepção, de forma a agregar as unidades calculadas até se chegar ao denominador (em UPs) característico absorvido por cada um destes $[8,14,18]$.
Por fim, o total dos esforços em UPs, que é igual à soma dos resultados obtidos, é capaz de retornar a mensuração do nível em que se encotra a estrutura de custos fabril, podendo-se assim quantificar economicamente a situação de qualqueir elementos que se encontram dentro dessa sistemática [19-20].

\section{COMPETITIVIDADE INDUSTRIAL}

O cenário atual das organizações tende para que elas se envolvam cada vez mais em ambientes altamente competitivos, seja em relação aos concorrentes diretos como para os indiretos [2,5]. Dessa forma, existe a predominante necessidade de se criar valor nas atividades desenvolvidas para a concepção de um produto ou serviço, tanto no âmbito externo quanto setorial e interno da organização, atendendo assim as expectativas dos consumidores, atraindo por consequência novos investimentos para a sua capacitação e aprimoramento [21-24].

Pode-se dizer, segundo pressupostos embasados por [2], que a competitividade setorial vai muito além da análise dos tradicionais adversários que atuam neste, pois esta depende de uma série de outras questões, chamadas de formas competitivas, que tangem desde os clientes até fornecedores, potenciais entrantes e possíveis substitutos, sendo agentes no comportamento dos vetores da lucratividade e do posicionamento estratégico da empresa [9, 25-26].

Em complemento, [27] comentam sobre o nível atual de competitividade através de dois pontos de vista complementares relacionados com o cliente: o consumo e a provisão, estando o primeiro relacionado à criação de valor por meio de fatores que não estão diretamente relacionados ao produto, e sim as atividades intermediárias realizadas para que este chegue até o destino final, o cliente, focando na sua experiência durante o seu contato com a empresa.

Já o segundo permeia questões relativas a disponibilização dos recursos para venda pela dualidade existente entre o fornecedor e a organização, por meio da correta realização das atividades de suprimento, atendendo aos requisitos de qualidade, prazo e quantidades determinadas, contribuindo na criação da vantagem competitiva para ambos [18, 22, 27]. 


\section{CLUSTERIZAÇÃO DE DADOS}

O problema de clusterização consiste da necessidade de se agrupar um conjunto de dados, localizados em uma base finita, em subdivisões, no qual estarão alocados conforme o grau de similaridade. Para tal, pode existir (problema de K-clusterização) ou não (problema de clusterização automática) um número já definido de clusters para o modelo [28-31].

Esse tipo de abordagem pode ser realizada sob diversos pontos óticos, de maneira que os mais perceptíveis são caracterizados como qualitativos ou quantitativos, onde a primeira é determinada partindo-se da natureza as quais se deseja realizar a verificação, enquanto a segunda é mais recomendada quando se trata de estudos sob ambientes considerados de alta complexidade e com grande volume de dados manipulados [32-35].

Atualmente, a aplicação dos problemas de clusterização são encontrados em diversas esferas de estudo, partindo-se das mais tradicionais, encontradas na informática, matemática e estatística, até as combinadas com outras demandas de utilização, como a proposta prática elaborada neste estudo [36].

\section{MÉTODOS MULTICRITÉRIOS DE APOIO A TOMADA DE DECISÃO}

Dentro das situações avaliadas no cotidiano organizacional, o processo de tomada de decisão é costumeiramente reconhecido como de alta complexidade, principalmente por ser necessária a definição de critérios que condicionem para a escolha das alternativas em consideração [37-41].

Assim, a abordagem multicritério tem por finalidade estabelecer o comportamento do desempenho em relação aos principais fatores inseridos ao contexto, conforme a suas definições intrínsecas [39, 4244]. Por possuir um caráter científico e também subjetivo, esse tipo de abordagem visa o tratamento das informações, desde que estas sintetizem de maneira coerente a mensuração, visando a produção de conhecimento e aumentando o entendimento a respeito do problema [45-48].

Em específico, a Análise Hierárquica de Processos (AHP) consiste na verificação, através da realização de julgamentos durante todos os níveis da estrutura hierárquica estudada, de quais alternativas elencadas são mais interessantes para o caso, gerando assim uma pré-ordem das opções a fim de facilitar na escolha a ser realizada pelos decisores [48, 50-51].

A proposta tem por premissa básica indicar a importância relativa existente entre cada um dos fatores pertencentes a um nível principal, até se chegar aos critérios submetidos para análises quantitativas a respeito do problema, hierarquizados por meio de comparações paritárias, de forma a transcrever como as mudanças das características nos níveis mais altos se distribuem e afetam direta ou indiretamente os respectivos subníveis [52-53].

A construção hierárquica do sistema visa o desenvolvimento do raciocínio lógico do pesquisador, buscando a definição do objetivo para o qual está sendo proposto, de modo a ocorrer a divisão da estrutura em níveis, onde cada fator relevante é responsável, respectivamente, pelos localizados diretamente abaixo deles, partindo inicialmente de um primeiro nível que compreende os ligados diretamente ao objetivo central estudado, até o último, composto pelas alternativas ao SMD em consideração [40, 52, 54].

Como a interação entre os eles ocorre de maneira paritária, existe a possibilidade da associação de valores quantitativos que representam a importância na relação entre cada um deles e reflete a sua situação em pares, o que gera a matriz representativa do fator estudado e, posteriormente, as taxas de substituição globais dos critérios em proporção ao objetivo [40, 50, 52].

\section{METODOLOGIA}

Para o desenvolvimento da aplicação existe uma série de passos que devem ser cumpridos, propostos através de treze operações, segundo mostra a Figura 1 , iniciando-se a partir do dimensionamento do local abordado e chegando-se ao final na realização dos cálculos propriamente ditos, sendo as técnicas utilizadas nas etapas intermediárias mostradas durante a etapa de desenvolvimento do estudo.

Para a coleta de dados, esta foi realizada pelo contato dos pesquisadores com os setores industriais da empresa que são considerados como fundamentais para desempenhar as fases da aplicação do método. 


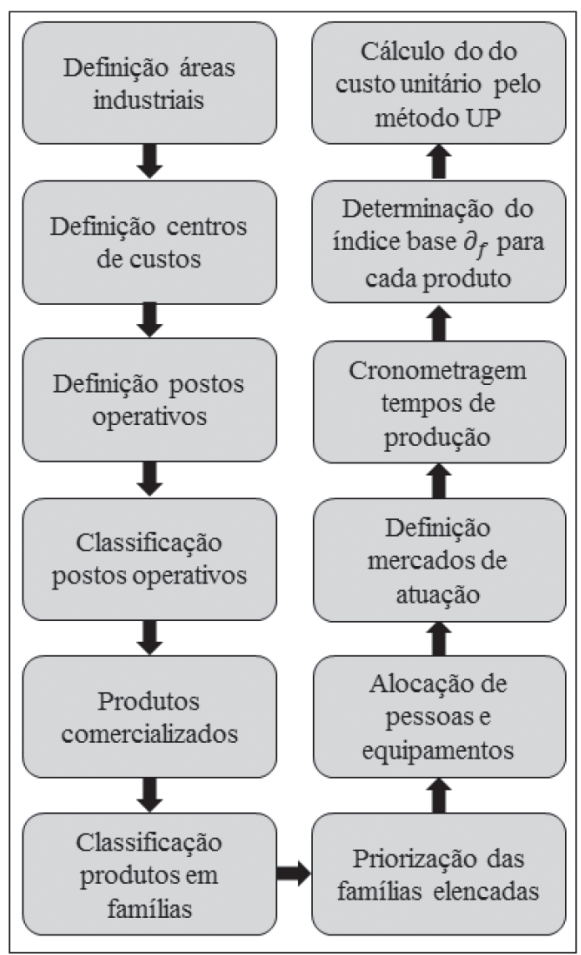

Figura 1. Etapas para a realização do custeio por UPs.

Cabe salientar que as informações geradas por este dimensionamento de processos estão propostas de maneira a serem inseridas em planilhas eletrônicas no formato do software Microsoft Excel®, onde é possível os cálculos necessários para a obtenção dos resultados esperados.

\section{DESENVOLVIMENTO DA MODELAGEM EM UM FRIGORÍFICO DE BOVINOS}

Diante da demanda predisposta, atualmente pode se afirmar que o frigorifico selecionado abate aproximadamente 11.500 bovinos por mês, com um total de 560 funcionários, formando um custo médio industrial por produto de $\mathrm{R} \$ 218,00$. Logo, o processo de verificação inicia-se com o dimensionamento das áreas na indústria a serem mensuradas, onde para o caso se elencou o Abate, a Produção, os Miúdos, o Charque e a Graxaria.

Mais especificadamente, após essa definição criaram-se os centros de custos ( $c c$ ) representativos dos locais onde ocorrem a derivação da matériaprima em produtos, seguindo a interrelação descrita esquematicamente pela Tabela 1, para um total de onze $c c$, dos quais $45 \%$ estão concentrados para a área considerada como chave para o processamento da carne que é a produção propriamente dita.

Tabela 1. Alocação dos custos por centro e áreas de fabricação.

\begin{tabular}{|l|c|l|}
\hline \multicolumn{1}{|c|}{ Centro de Custos } & cc & \multicolumn{1}{c|}{ Área } \\
\hline Mangueiras & 1 & Abate \\
\hline Abate & 2 & Abate \\
\hline Resfriamento de carcacas & 3 & Abate \\
\hline Sala de corte & 4 & Produção \\
\hline Desossa & 5 & Produção \\
\hline Embalagem & 6 & Produção \\
\hline Camara de resfriados & 7 & Produção \\
\hline Camara de congelados & 8 & Produção \\
\hline Miudos & 9 & Miúdos \\
\hline Charque & 10 & Charque \\
\hline Graxaria & 11 & Graxaria \\
\hline
\end{tabular}

Com isso, parte-se para as etapas de determinação dos postos operativos e sua recorrente classificação, onde para o primeiro são especificados os locais em que ocorrem os esforços da manufatura, separados em vinte e nove partes $a, \forall a \in\{1,2, \ldots, k\}$, enquanto o segundo está estabelecido de acordo com o(s) tipo(s) de esforços que ocorre no posto, sendo classificados para tanto em seis maneiras distintas $(g), \forall g \in\{1,2, \ldots, r\}$, de acordo com as suas características no processo em questão: máquina, manual, transporte, retrabalho, qualidade e o Serviço de Inspeção Federal (SIF).

Estas condições estão dispostas seguindo os preceitos descritos pelo método de clusterização hierárquica, no qual busca-se realizar a organização dos dados disponibilizados sob a ótica top-down (fato característico da problemática abordada nesta etapa), possuíndo inicalmente um grupo undimensional que se subdivide, ao longo de etapas em grupos, até se chegar ao último nível capaz de retornar com maior número de detalhes esperados para a classificação em questão, evitando o efeito da subjetividade no momento da realização desta atividade.

Para tanto, parte-se inicialmente da definição do número de níveis percorridos até se chegar ao detalhamento esperado, havendo a segregação por meio da similaridade existente entre as partes e a natureza através da caracterização quantitativas de cada uma destas e calculadas em pares a partir 
das definições propostas por [28], de modo a ser possível encontrar a distribuição dos elementos de acordo com a Tabela 2.

Desta forma, a clusterização foi um meio para facilitar o proceso de agrupamento dos postos operativos, de modo a serem estudados em conjuntos similares, e não individualizados.

Ainda as classificações denominadas por "Retrabalho" e "Qualidade" não possuem postos operativos elencados, bem como os denominados por "Máquina" e "Manual" foram classificados predominantemente para a ordenação, com mais de $82 \%$ dos pontos constatados.

Tabela 2.Clusterização dos Postos Operativos.

\begin{tabular}{|l|c|c|}
\hline Classificação & $g$ & Total \\
\hline Máquina & 1 & 11 \\
\hline Manual & 2 & 13 \\
\hline Transporte & 3 & 4 \\
\hline Retrabalho & 4 & 0 \\
\hline Qualidade & 5 & 0 \\
\hline SIF & 6 & 1 \\
\hline
\end{tabular}

Com isso, é necessário elencar os produtos que fazem parte do portfolio de comercialização. Por se tratar atualmente de um número superior a 300 tipos distintos, a fim de facilitar a verificação, foi proposto o arranjo destes em famílias, tomando por base o método de clusterização matricial relacionado a dois fatores característicos: a matéria-prima de origem e a forma de comercialização, encontrandose sete formas de classificação distintas $(f), \forall f \in$ $\{1,2, \ldots, n\}$, capazes de compreender toda a gama de produtos da empresa, sendo elas: Coxa $(f=1)$, Alcatra $(f=2)$, Lombo $(f=3)$, Paleta $(f=4)$, Agulha $(f=5)$, Costilhar $(f=6)$ e Outros $(f=7)$.

Seguindo a estrutura de escolhas desenvolvida por [55] e devido ao grau de afinidade atingido com resultados obtidos em relação a realidade do estudo, definiu-se para a determinação das principais a utilização dos Processos de Análise Hierárquica Referenciado e B-G, ao qual se propõe o julgamento da importância relativa de cada um delas por um comparativo par a par, sendo uma vantagem relativamente elevada em proporção aos demais métodos multicritério. Para tanto, considerou-se no caso a utilização de dois fatores $d \in\left\{F p_{f}, R_{f}\right\}$ preponderantes: o faturamento bruto proporcional $\left(F_{p f}\right)$ e o rendimento médio de carne por boi $\left(R_{f}\right)$.

Quanto ao grau de priorização destas em comparação aos fatores elencados, é proposta a elaboração das matrizes $C_{d_{n * n}}=\left(c_{i j}\right)$, seguindo os pressupostos de [40] e exposta pela Matriz (1),

$$
C_{d_{n \cdot n}}=\left[\begin{array}{cccc}
1 & c_{12} & \cdots & c_{1 n} \\
1 / c_{12} & 1 & \cdots & c_{2 n} \\
\vdots & \vdots & \ddots & \vdots \\
1 / c_{1 n} & 1 / c_{2 n} & \cdots & 1
\end{array}\right]
$$

tal que $c_{i j}=c ; c_{j i}=1 / c, \forall c_{i j} * c_{j i}=c_{i n}, j=1,2, \ldots, n e$ $c_{i i}=1$, além do número de julgamentos necessários para a construção de cada uma é definido por $\mathrm{n}^{*}(\mathrm{n}-1) / 2$, de maneira que $n$, nesta situação, compreende o número total de elementos $c_{i j}$ existentes na matriz. Com isso, devem ser atribuídos para cada família os valores de $f$ em analogia ao fator $d$ determinante no momento do cálculo, caracterizando quantitativamente a sua importância e possibilitando a busca dos vetores em semelhança ao conjunto de fatores constituintes da matriz $\left(\bar{v}_{f}\left(C_{d_{n^{*} n}}\right)\right)$, normalizados por intermédio do vetor $\bar{v}_{P_{f}}\left(C_{d_{n^{*} n}}\right)$ para com o somatório dos resultados estabelecidos para todas elas, conforme mostra a equação (1), baseado nos pressupostos de [38],

$$
\bar{v}_{P_{f}}\left(C_{d_{n^{*} n}}\right)=\frac{\bar{v}_{f}\left(C_{d_{n^{*} n}}\right)}{\sum_{i=1}^{n} \bar{v}_{f}\left(C_{d_{n^{*} n}}\right)}
$$

de maneira que o $\sum_{i=1}^{n} \bar{v}_{f}\left(C_{n^{*} n}\right)=1$, além do cálculo da importância relativa entre as alternativas $\left(w_{f}\right)$, através da comparação da estimativa para a importância dos fatores $\left(x_{d}\right)$ previamente definidos ao estudo e o vetor de normalização da matriz $\bar{v}_{P_{f}}\left(C_{d_{n^{*} n}}\right)$, resultando na proposta descrita pela equação (2).

$$
w_{f} \sum_{d=F p_{f}}^{R_{f}} x_{d} * \bar{v}_{P_{f}}\left(C_{d_{n * n}}\right)
$$

Para a análise B-G, primeiramente deve-se encontrar, dentro de cada matriz $C_{d_{n^{*} n}}$, o maior $\bar{v}_{i}\left(C_{d_{n^{*} n}}\right)$, denominado por $z_{\text {maxd }}$, de modo a tornar este proporcional a uma unidade de medida, possibilitando com isso a obtenção dos elementos $z_{i d}$ proporcionais 
ao máximo, chegando-se finalmente ao valor da importância de cada fator $\left(\delta_{d}\right)$ em relação ao modelo estabelecido e, consequentemente, o cálculo pela equação (3) da relativa priorização existente para as famílias $\left(\mu_{i}\right)$.

$$
\mu_{i}=\sum_{d=F p_{f}}^{R_{f}} \delta_{\mathrm{d}} * \mathrm{z}_{i d}
$$

Por fim, pelos dois métodos é possível se obter a média dos valores $\left(\rho_{f}\right)$ nos quais se estabelecerá a pré-ordem das famílias selecionadas, bem como das diferenças entre eles $\left(\Delta_{f}\right)$, verificando o nível de discrepância dos resultados para o caso, conforme as equações (4) e (5).

$$
\begin{gathered}
\rho_{f}=\frac{\left(\mu_{\mathrm{i}}+\mathrm{w}_{\mathrm{f}}\right)}{p} \\
\Delta_{f}=\mu_{i}-w_{f}
\end{gathered}
$$

A Tabela 3 mostra a ordenação obtida das famílias de acordo com a sua relevância mensurada, chegandose ao consenso de que a família denominada por "Coxa" é a mais relevante para o sistema em questão.

Para a fase de cadastramento e alocação das pessoas e dos equipamentos é necessário se elencar os dispêndios com o salário de cada colaborador e o consumo de energia elétrica, depreciação, vida útil, peças de manutenção e tempo de manutenção médio empregado mensalmente dos maquinários, de acordo com cada um dos grupos operativos classificados anteriormente. No caso de haver algum recurso humano ou material que seja dividido em dois ou mais desses, buscou-se alocar este no que possui a maior demanda para o período de verificação.

Tabela 3.Resultado final da ordenação estabelecida para as famílias de produtos.

\begin{tabular}{|l|c|c|c|c|}
\hline \multicolumn{1}{|c|}{$\boldsymbol{f}$} & $\boldsymbol{w}_{\boldsymbol{f}}$ & $\rho_{f}$ & $\Delta_{f}$ & $\rho_{f}$ \\
\hline Coxa & $17,9 \%$ & $17,0 \%$ & $0,9 \%$ & $17,4 \%$ \\
\hline Alcatra & $12,2 \%$ & $12,8 \%$ & $-0,6 \%$ & $12,5 \%$ \\
\hline Lombo & $13,1 \%$ & $13,0 \%$ & $0,0 \%$ & $13,1 \%$ \\
\hline Paleta & $15,2 \%$ & $15,2 \%$ & $0,0 \%$ & $15,2 \%$ \\
\hline Agulha & $15,8 \%$ & $15,3 \%$ & $0,5 \%$ & $15,5 \%$ \\
\hline Costilhar & $17,3 \%$ & $17,8 \%$ & $-0,5 \%$ & $17,5 \%$ \\
\hline Outros & $8,5 \%$ & $8,9 \%$ & $-0,4 \%$ & $8,7 \%$ \\
\hline
\end{tabular}

Após, surge a necessidade da definição dos mercados de atuação para cada família, embasada a partir do conhecimento sobre a legislação fiscal aplicada para o tipo de comercialização em questão, pois a tributação realizada entre os produtos é diferenciada conforme o seu destino de consumo.

Com todo cadastramento da indústria realizado, definitivamente entra-se nas mensurações do tempo necessário $\left(D_{f_{\gamma}}\right)$ para que os processos possam ser realizados por quilo de carne para cada família ( $\mathrm{s} / \mathrm{kg}$ ou $\mathrm{h} / \mathrm{kg}$ ), derivados dos valores encontrados para os esforços operativos $D_{g \gamma}$ que resultaram da mensuração de realizada em cada processo operativo, conforme a relação descrita pela equação (6),

$$
D_{f_{\gamma}}=\sum_{g=1}^{r} D_{g_{\gamma}} \rightarrow D_{g_{\gamma}}=\sum_{a=1}^{k} D_{a_{\gamma}}
$$

$\forall \gamma \in\{b, 1,2, \ldots, n\}$ representativo do período no qual a mensuração está sendo submetida para verificação, considerando $b$ como o período base que contém os valores considerados como ideais na gestão dos custos. Com esta determinação é necessário, em específico, mensurar a estimativa do tempo padrão gasto para a manufatura de cada uma dessas tarefas no fluxo industrial, seja em função do esforço da máquina $\left(\overline{E M_{g_{\gamma}}}\right)$ ou do trabalho humano $\left(\overline{E O_{g}}\right)$, segundo descrito pela equação (7) em função da quantidade de quilos de carne produzidos $Q p_{g_{\gamma}}$ na tarefa em questão (em kg),

$$
D_{g_{\gamma}}=\left\{\begin{array}{l}
{\left[\left(\frac{\overline{E M}}{Q p}\right)_{g}\right]_{\gamma} \rightarrow Q p_{g_{\gamma}}=\overline{Q_{t \gamma}} * R_{D_{g}} \geq 0} \\
{\left[(\overline{E O})_{g}\right]_{\gamma}}
\end{array}\right.
$$

no qual $\bar{Q}_{t_{\gamma}}$ representa a média do peso total por bovino manufaturado $(\mathrm{kg})$ e $R_{D_{g}}$ é o rendimento estimado para a atividade em questão. Com a execução da cronometragem dos procedimentos industriais, notou-se principalmente a obtenção de baixos resultados para as operações que envolvem a utilização de máquinas no processo produtivo, o que já se tornou, em um primeiro instante, um ponto de relativa observação por parte dos dirigentes. 
A partir dessas definições e com a cronometragens dos tempos de cada processos $D_{a_{\gamma}}$ realizada, tornase plausível a determinação do índice base $\partial_{f_{\gamma}}$ relativo a mensuração dos dispêndios financeiros de fabricação das famílias de produtos, partindo inicialmente da definição do indicador padrão $\partial_{p_{\gamma}}$ em relação ao tempo padrão $D_{p_{\gamma}}$ para os esforços produtivos, servindo como balizador no momento de se atribuir uma contagem de UP padrão e dos índices de todos os restantes $\left(\partial_{a_{\gamma}}\right)$ conforme segue a equação (8):

$$
D_{p_{\gamma}} \propto \partial_{p_{\gamma}}\left\{\begin{array}{c}
D_{a_{\gamma}}>D_{p_{\gamma}} \rightarrow \lim _{D_{a_{\gamma}} \rightarrow \infty} \partial_{a_{\gamma}}=\infty \\
D_{a_{\gamma}}<D_{p_{\gamma}} \rightarrow \lim _{D_{a_{\gamma}} \rightarrow 0} \partial_{a_{\gamma}}=0 \\
D_{a_{\gamma}}=D_{p_{\gamma}} \rightarrow \partial_{p_{\gamma}}=10
\end{array}\right.
$$

Para o caso foi considerado como padrão $D_{p_{\gamma}}=0,0233 \mathrm{~h} / \mathrm{kg}$, que corresponde ao total de $\partial_{p_{\gamma}}=10$ UP's, sendo possível afirmar que o processo que demorar mais ou menos tempo na fabricação terá um valor proporcional de UPs disponíveis para o custeamento, entendendo que a função tempo passou a ser o único indexador de unidade de medida, eliminando as chances de haver divergências nas mensurações, parao $\partial_{f_{\gamma}}$ obtido na equação (9),

$$
\partial_{f_{\gamma}}=\sum_{g=1}^{r} \partial_{g_{\gamma}} \rightarrow \partial_{g_{\gamma}}=\sum_{a=1}^{k} \partial_{a_{\gamma}}
$$

A partir desse ponto são coletados todos os gastos nos centros de custos da indústria $\left(C l_{t_{\gamma}}\right)$, equação (10),e a proporção relativa de UPs $P p_{f_{\gamma}}$ necessária para a manufatura em questão, equação (11), para se obter o resultado individual dos produtos $\left(C U_{f_{\gamma}}\right)$ proposta pela equação (12),

$$
\begin{gathered}
C I_{t_{\gamma}}=\sum_{c c=1}^{n}\left[\left(C p^{*} Q p\right)_{c c}\right]_{\gamma} \\
P p_{f_{\gamma}}=\left(\frac{\partial_{f}}{\partial_{t}}\right)_{\gamma} \rightarrow \partial_{t_{\gamma}}=\sum_{f=1}^{n} \partial_{f_{\gamma}} \\
C U_{f_{\gamma}}=P p_{f_{\gamma}} * C I_{t_{\gamma}}
\end{gathered}
$$

onde $C p_{c c}$ é relativo ao gasto por quilo de carne processada e $Q p_{c c_{\gamma}}$ é a respectiva quantidade processada naquele local. Por fim, chega-se ao total necessário por quilo para a produção, de modo que a equação (13) descreve, partindo-se das definições de custo unitário $\left(C U_{f_{\gamma}}\right)$ e da quantidade de carne disponível para a sua produção $\left(Q p_{f_{\gamma}}\right)$, sendo este variável conforme a qualidade do produto e a maneira com que é realizada a comercialização junto aos produtores rurais.

$$
C Q_{f_{\gamma}}=\left[\left(\frac{C U}{Q p}\right)_{f}\right]_{\gamma}
$$

Com essa e as demais resoluções propostas durante a execução das etapas do trabalho é possível obter o cenário em que a empresa se encontra, segundo a Tabela 4 correspondente ao cálculo das veriáveis em um cenário considerado como ideal para cada uma das famílias, denominado por base, definido como padrão de comparação da situação de cada um dos meses seguintes elencados para estudo durante o ano de 2012.

Dessa forma, percebe-se que ao final gerou-se um montante total $\partial_{f_{b}}$ igual a $\mathrm{R} \$ 1.325,29$ para transformar um boi em material acabado proporcional a $C Q_{f_{b}}=\mathrm{R} \$ 0,65$ e $C U_{f_{b}}=\mathrm{R} \$ 143,26$.

Tabela 4.Rateio do custo industrial atraves do esforço de produção.

\begin{tabular}{|c|l|c|c|c|c|c|}
\hline $\boldsymbol{f}$ & Famílias & $\begin{array}{c}Q p_{f_{\gamma}} \\
(\mathbf{k g})\end{array}$ & $\begin{array}{c}\partial_{f_{\gamma}} \\
(\mathbf{U P})\end{array}$ & $\begin{array}{c}P p_{f_{\gamma}} \\
(\mathbf{\%})\end{array}$ & $\begin{array}{c}C U_{f_{\gamma}} \\
(\mathbf{R} \mathbf{\$})\end{array}$ & $\begin{array}{c}C Q_{f_{\gamma}} \\
(\mathbf{R} \mathbf{/ k g})\end{array}$ \\
\hline 1 & Coxa & 57,0 & 247,1 & 18,6 & 26,71 & 0,47 \\
\hline 2 & Alcatra & 14,1 & 116,1 & 8,7 & 12,55 & 0,88 \\
\hline 3 & Lombo & 29,6 & 169,0 & 12,7 & 18,27 & 0,62 \\
\hline 4 & Paleta & 33,6 & 229,0 & 17,2 & 24,76 & 0,74 \\
\hline 5 & Agulha & 45,4 & 223,4 & 16,8 & 24,15 & 0,53 \\
\hline 6 & Costilhar & 29,0 & 280,7 & 21,1 & 30,34 & 1,05 \\
\hline 7 & Outros & 11,0 & 59,6 & 4,50 & 6,45 & 0,59 \\
\hline & Total & 220 & 1325 & 100 & 143,26 & 0,65 \\
\hline
\end{tabular}

Quanto aos resultados específicos, notou-se que o "Costilhar" é o maior fator oneroso para a manufatura $\left(C Q_{6_{b}}=\mathrm{R} \$ 1,05\right)$, pois possui o índice base mais elevado dentre todos, devido principalmente tempo necessário para o desenvolvimento dos seus processos $\left(\partial_{6_{b}}=280,7 \mathrm{UP}\right), 12 \%$ superior ao segundo maior, apesar do rendimento para o total de carne aproveitada no boi ser apenas a quinta colocada $\left(Q p_{6_{b}}=29,0 \mathrm{~kg}\right)$, somado ao obtido para 
o gasto absoluto $\left(C U_{6_{b}}=\mathrm{R} \$ 30,34\right)$, relativo ao encontrado em UPs com relação ao tempo gasto para a fabricação dos seus conponentes.

Ainda, destaca-se para esse estudo o alto custo por quilo encontrado para a "Alcatra" $\left(C Q_{2_{b}}=\mathrm{R} \$ 0,88 /\right.$ $\mathrm{kg}$ ), de modo que, apesar do baixo valor encontrado em $C U_{2 b}(\mathrm{R} \$ 12,55)$, este é considerado como elevado quando observada a quantidade produzida por boi $\left(Q p_{2_{b}}=14,1 \mathrm{~kg}\right)$, chegando-se a conclusão que esse corte deve ser comercializado através de produtos com um maior valor no preço médio repassado ao consumidor, buscando a redução desses efeitos.

A seguir tem-se em terceiro lugar, tanto para o gasto tanto no custo por produção, a "Paleta", com $C Q_{4_{b}}=\mathrm{R} \$ 0,74$ e $C U_{4_{b}}=\mathrm{R} \$ 24,76$, respectivamente, devido aos altos resultados encontrados para o aproveitamento de carne $\left(Q p_{4_{b}}=33,6 \mathrm{~kg}\right)$ e UP da produção $\left(\partial_{4_{b}}=229,0 \mathrm{UP}\right)$.

De modo geral, para a estimativa dos períodos base proposta pela variável $\bar{Q}_{t_{b}}$, pode-se afirmar que os $C Q_{f_{b}}$ variaram com uma amplitude de $\mathrm{R} \$ 0,58$, $81 \%$ inferior ao máximo $(\mathrm{R} \$ 1,05)$ e $9 \%$ superior ao mínimo estabelecido ( $\mathrm{R} \$ 0,53)$, enquanto para o $C U_{f_{b}}$ médio é igual a $\mathrm{R} \$ 20,46,48 \%$ abaixo do máximo e $68 \%$ maior que o mínimo, caracterizado por ser de baixa variação dos extremos para ambos indicadores. Com isso parte-se a próxima etapa de aplicação dos indicadores para a mensuração mês a mês, tomando por referência seis meses, desde Março até Agosto do ano de 2012, obtendo-se as estimativas gerais mostradas na Tabela 5 .

Tabela 5.Resultados mensurados dos custos industriais de março a agosto/2012.

\begin{tabular}{|c|c|c|c|c|}
\hline Indicador & $\begin{array}{c}\text { Base } \\
(\gamma=b)\end{array}$ & $\begin{array}{c}\text { Março } \\
(\gamma=1)\end{array}$ & $\begin{array}{c}\text { Abril } \\
(\gamma=2)\end{array}$ & $\begin{array}{c}\text { Maio } \\
(\gamma=3)\end{array}$ \\
\hline$\partial_{f_{\gamma}}(\mathrm{UP})$ & 1325,2 & 1416,0 & 1381,7 & 1406,4 \\
\hline$C U_{f_{\gamma}}(\mathrm{R} \$)$ & 143,26 & 146,20 & 149,77 & 129,06 \\
\hline$Q p_{f_{\gamma}}(\mathrm{kg})$ & 220,0 & 235,0 & 229,3 & 233,4 \\
\hline$C Q_{f_{\gamma}}(\mathrm{R} \$ / \mathrm{kg})$ & 0,65 & 0,62 & 0,65 & 0,55 \\
\hline Indicador & $\begin{array}{c}\text { Base } \\
(\gamma=b)\end{array}$ & $\begin{array}{c}\text { Junho } \\
(\gamma=4)\end{array}$ & $\begin{array}{c}\text { Julho } \\
(\gamma=5)\end{array}$ & $\begin{array}{c}\text { Agosto } \\
(\gamma=6)\end{array}$ \\
\hline$\partial_{f_{\gamma}}(\mathrm{UP})$ & 1325,2 & 1393,7 & 1385,2 & 1401,6 \\
\hline$C U_{f_{\gamma}}(\mathrm{R} \$)$ & 143,26 & 153,74 & 145,78 & 134,20 \\
\hline $\bar{Q}_{t_{\gamma}}(\mathrm{kg})$ & 220,0 & 231,3 & 229,9 & 232,6 \\
\hline$C Q_{f_{\gamma}}(\mathrm{R} \$ / \mathrm{kg})$ & 0,65 & 0,66 & 0,63 & 0,58 \\
\hline & & & &
\end{tabular}

Para as estimativas unitárias $C Q_{f_{\gamma}}$ é possível notar que foram obtidos valores satisfatórios para quatro dos seisperíodos (Março, Maio, Julho e Agosto) avaliadosem relação ao valor estipulado como ideal $\left(C Q_{f_{b}}=\mathrm{R} \$ 0,65\right)$, sendo os melhores resultados obtidos para os meses de Maio $\left(C Q_{f_{3}}=\mathrm{R} \$ 0,55\right) \mathrm{e}$ Agosto $\left(C Q_{f_{3}}=\mathrm{R} \$ 0,58\right)$, demonstrando assim a boa situação da empresa e a possibilidade de se obter valores mais reduzidos para os próximos meses, apesar do desempenho encontrado para o mês de Junho $\left(C Q_{f_{4}}=\mathrm{R} \$ 0,66\right)$ que, mesmo sendo considerado relativamente fraco em relação aos melhores se encontra próximo do considerado como ideal.

Quanto a $\bar{Q}_{t,}$, estes estão predispostos conforme ao comportamento da demanda do mercado agropecuário para cada período, independendo da ação direta das modificações estruturais, notando-se que todos os resultados localizados acima da estimativa base.

É importante destacar que por melhor que tenham sido os resultados encontrados para o sistema, os valores dos custos de produção em quatro dos seis períodos (Março, Abril, Junho e Julho) atingiu patamares superiores ao estipulado como base $\left(C U_{f_{b}}=\mathrm{R} \$ 143,26\right)$, fato que contrapõe os bons resultados demonstrados anteriormente e considerada por excessão somente nos meses em que se encontrou os melhores valores para o custo unitário de produção (Maio e Agosto).

Para os valores encontrados para a quantidade de carne média disponibilizada para a produção, tem-se que todos os meses superaram as expectativas previamente realizadas, cadendo um destaque especial para os períodos de Março $\left(Q p_{f_{1}}=235,0 \mathrm{~kg}\right)$ e Maio $\left(Q p_{f_{3}}=233,4 \mathrm{~kg}\right)$.

Portanto, diante dos pressupostos estabelecidos pelas variáveis mostradas pode-se se afirmar que apesar de haver um significativo aumento dos custos de produção na maioria dos períodos designados para o estudo, este pode ser explicado pela necessidade de aumento na demanda de manufatura de carne, de maneira que esse comportamento está diretamente vinculado a necessidade de uma maior capacidade de produção para atender tal variação.

\section{CONCLUSÃO}

Com a aplicação do sistema de informações apresentado, a empresa conseguiu reunir esforços 
de todas as suas áreas em prol da redução dos seus custos, o que transformou o método UP em numa ferramenta de trabalho rotineira e bem aceita pelos colabororadores, pois possibilitou uma visão detalhada principalmente dos esforços humanos aplicados na manufatura.

Além disso, permitiu a visualização de maneira mais clara de soluções rápidas e eficientes sobre qualquer alteração que venha a ser realizada, oferecendo assim subsídios para que se alcance um número de abates e cabeças de gado considerado ideal para o momento, no caso $\left(C Q_{f_{b}}=\mathrm{R} \$ 0,65\right)$, tornando possível um aumento do poder sobre o próprio custo para enfrentar as condições que o mercado.

Diante desse e de outros fatos, pode-se afirmar que o objetivo proposto inicialmente foi atingido com êxido pelos pesquisadores, principalmente no que tange a questão de se mensurar o quanto cada família de produtos influencia nas finanças industriais de uma empresa frigorífica, podendo-se assim abrir possiblidades de melhorias sob diversos cortes de tempos distintos, auxiliando assim no processo de apoio para a tomada das melhores decisões a respeito da gestão estratégica para os cenários estudados.

Cabe salientar que este é o início de uma etapa, pois com a implantação de um modelo de medição de desempenho através do sistema de custeio UP no frigorífico em questão, os trabalhos tendem a continuar, visando a aprimorar as avaliações em outras áreas chave, com novos métodos e procedimentos que possam contribuir nos resultados da empresa.

Em conjunto, o presente trabalho transformou a proposta de medição de desempenho organizacional, juntamente com o sistema UP, em uma ferramenta de trabalho importante para a indústria de cortes de gado, pois, além de medir os esforços para a produção de cada produto, eles permitem uma visão detalhada dos esforços e custos desprendidos em cada centro de custo, possibilitando, assim, soluções mais rápidas e eficientes sobre qualquer alteração desejada, de modo a fomentar novos estudos teóricos e práticos a respeito do assunto.

Como limitações da pesquisa, se verificou inicialmente a falta de documentos e dados organizados na empresa a respeito de custeio da produção, o que tornou o trabalho desgastante e, muitas vezes, repetitivo até se chegar a consolidação destes em informações claras e bem definidas a respeito do comportamento do sistema.

Como expectativas futuras, espera-se que o método possa ser aplicado a outras empresas do setor frigorífico, seja bovino ou de outra natureza de corte, de modo a poder se realizar a comparação entre os resultados obtidos nesta empresa com as demais a serem pesquisadas. Outro ponto observado como possibilidade futura é a utilização do método em outros tipos de indústria, a fim de verificar qual é o seu nível de adaptabilidade a outras formas de produção industrial.

\section{REFERÊNCIAS}

[1] N. Slack, R. Chambers, R. Johnston and A. Betts. "Operation and process management: principles and practice for strategic impact". Prentice Hall. Lebanon. 2008.

[2] M. Porter. "Competitividade". Campus. São Paulo, Brasil. 2009.

[3] F. Allora e V. Allora. "UP: Unidade de Produção". Pioneira. São Paulo, Brasil. 1995.

[4] M. Anderson, O. Asdemir and A. Tripathy. "Use of precedente and antecedente information in strategic cost management". Journal of Business research. Vol. $66 \mathrm{~N}^{\circ} 5$, pp. 643-650. 2013.

[5] P.F. Drucker and J.A. Marciariello. "The effective executive in action". Harper Business. New York, Estados Unidos. 2005.

[6] D. Grahovac and V. Devedzic. "Comex: a cost management expert system". Expert Systems with Applications. Vol. 37 N $^{\mathrm{o}} 12$, pp. 7684-7695. 2011.

[7] E. Martins. "Contabilidade de custos". Atlas. São Paulo, Brasil. 2003.

[8] E. Martins e W. Rocha. "Métodos de custeio comparados". Atlas. São Paulo, Brasil. 2010.

[9] K. Phusavat, N. Comepa, A. Sitko-Lutek and K. Ooi. "Intellectual capital: national implications for industrial competitiveness". Industrial Management \& Data Systems. Vol. $112 \mathrm{~N}^{\circ}$ 6, pp. 866-890. 2012.

[10] H.F. Mella and I.D. Bravo. "Análisis de los factores determinantes de la calidad percibida del servicio prestado por una cooperativa de ahorro y crédito: una aplicación basada en modelos de ecuaciones estructurales". 
Ingeniare. Revista Chilena de Ingeniería, Vol. $21 \mathrm{~N}^{\circ}$ 2, pp. 232-247. 2013.

[11] A.L. Neuenfeldt Júnior, J.C.M. Siluk, M. Soliman and K.F.S. Marques. "Study to evaluate the performance development of Brazilian franchise segments". Independent Journal of Management \& Production, Vol. 5 $\mathrm{N}^{\circ}$ 2, pp. 381-397. 2014.

[12] R.P. Costa, J.C.M. Siluk, A.L. Neuenfeldt Júnior, M. Soliman, S.R. De Paris e V.D. Cattelan. "A gestão da competitividade industrial por meio da aplicação dos métodos UP e multicritério no setor frigorífico de bovinos". II Fórum Internacional Ecoinovar. Santa Maria, Brasil. 2013.

[13] L.H. Rodrigues and G. Brady. "Cost accounting and production control in a multiproduct effort method". International Journal of Operations \& Production Management. Vol. $12 \mathrm{~N}^{\mathrm{o}} 10$, pp. 66-81. 1992.

[14] E. Chacon, I. Besembel, D.M. Rivero and J. Cardillo. "Embedded holonics systems in production process: holonicunit of production. Revista Técnica de la Facultad de Ingeniería Universidad del Zulia. Vol. $32 \mathrm{~N}^{\circ}$ 1, pp. 3-13. 2009.

[15] V. Oarga. “'Controlling' as a unit of production planning and control". The Romanian Economic Journal. Vol. XII No 34, pp. 61-83. 2009.

[16] R.L. Cardoso. "Contabilidade Gerencial". Atlas. São Paulo, Brasil. 2007.

[17] F. Wrublel, C.A. Diehl, L.A. Tolgo e E. Ott. "Uma proposta para a validação de categorias sobre gestão estratégica de custos". Revista Brasileira de Gestão de Negócios. Vol. 13 $\mathrm{N}^{\circ}$ 40, pp. 332-348. 2011.

[18] Q.A. Malik, I. Saif, N. Safwan and A. Gulzar. "Impact of organizational innovation on success of cost management techniques in value creation: evidence from manufacturing sector of Pakistan". African Journal of Business Management. Vol. $5 \mathrm{~N}^{\circ} 15$, pp. 66186624. 2011.

[19] G. Gantzel e V. Allora. "Revolução nos Custos". Editora Casa da Qualidade. Itaigara, Brasil. 1996.

[20] F.T. Sakamoto, T.C. Frederico, V. Allora e S. Espindola. "Equipes multidisciplinares no desenvolvimento de sistemas de custeio diante da atual necessidade de informação para tomada de decisão". VIII Congresso del instituto internacional de costos. Punta del Este, Uruguai. Novembro de 2003.

[21] E. Zogbi. "Competitividade através da gestão da inovação". Atlas. São Paulo, Brasil. 2008.

[22] P. Zhang and K. London. "Towards an internationalized sustainable industrial competitiveness model". Competitive Review: An International Business Journal. Vol. 23 No 2, pp. 95-113. 2013.

[23] J.Q. Silveira, J.C.C.B.S. Mello e L. AnguloMeza. "Evaluación de la eficiencia de las compañías aéreas brasileñas a través de un modelo híbrido de análisis envolvente de datos (DEA) y programación lineal multiobjectivo". Ingeniare. Revista chilena de ingeniería. Vol. $20 \mathrm{~N}^{\circ}$ 3, pp. 331-342. 2012.

[24] T.F. Scarano, J.C.M. Siluk, E.O.B. Nara, A.L. Neuenfeldt Júnior e F.B.B. Da Fontoura. "Diagnóstico do desempenho organizacional em empresas do setor metal mecânico". Espacios. Vol. 35 N $^{\circ}$ 3, p. 18. 2014.

[25] L.C. Di Serio e M.A. Vasconcellos. "Estratégia e competitividade empresarial: inovação e criação de valor". Saraiva: São Paulo, Brasil. 2009.

[26] A.P.B. Bartz, A.D. Weise e J.E.Ruppenthal. "Aplicação da manufatura enxuta em uma indústria de equipamentos agrícolas". Ingeniare. Revista chilena de ingeniería. Vol. $21 \mathrm{~N}^{\circ}$ 1, pp. 147-158. 2013.

[27] J.P. Womack e D.T. Jones. "Soluções enxutas". Elsevier. Rio de Janeiro, Brasil. 2006.

[28] P. Berkhin. "Survey of clustering data mining techniques". Technical report. Accrue Software. 2002.

[29] D. Fasulo. "An analysis of recent work on clustering algorithms". Technical report. Dept. of Computer Science and Engineering, Univ. of Washington, 1999.

[30] D. Ienco, R.G. Pensa and R. Meo. "From context to distance: learning dissimilarity for categorical data clustering". ACM Transactions on Knowledge Discovery. Vol 6 N$^{\circ}$ 1. 2012.

[31] X. Yan, Y. Zhu, W. Zou and L. Wang. "A new approach for data clustering using hybrid artificial bee colony algorithm". Neurocomputing. Vol. 97, pp. 241-250. 2012.

[32] A.K. Jain and R.C. Dubes. "Algorithms for clustering data". Prentice Hall. New Jersey, Estados Unidos. 1998. 
[33] A.K. Jain. "Data clustering: 50 years beyond K-means". Pattern Recognition Letters. Vol. 31 No 8, p. 651-666. 2010.

[34] R.M.C.R. Souza and F.A.T. Carvalho. "Clustering of an interval data base on cityblock distances". Pattern Recognition Letters. Vol. $25 \mathrm{~N}^{\circ}$ 3, pp. 353-365. 2004.

[35] M. Taherdangkoo, M. Yazdi and M.H. Bagheri. "A powerful and efficient evolutionary optimization algorithm based on stem cells algorithm for data clustering". Central European Journal of Computer Science. Vol. 2 $\mathrm{N}^{\circ}$ 1, pp. 47-59. 2012.

[36] D.R. Ludewig, M.A.U. Opazo, R.M.T. Gimenes e E.G. Souza. "O processo de gestão de custos e planejamento de resultados utilizando técnicas de análise estatística de agrupamentos". Acta Scientiarum Technology. Vol. $31 \mathrm{~N}^{\circ}$ 2, pp. 215-220. 2009.

[37] A.T. Almeida e A.P.C.S. Costa. "Aplicações com métodos multicritério de apoio à decisão". Editora Universitária. Recife, Brasil. 2003.

[38] C.F. Gomes e L.F.A.M. Gomes. "Tomada de decisão gerencial: enfoque multicritério". Atlas. São Paulo, Brasil. 2012.

[39] L.F.A.M. Gomes, M.C.G. Araya e C. Carignano. "Tomada de decisões em cenários complexos: Introdução aos métodos discretos de apoio multicritério à decisão". Cengage Learning. São Paulo, Brasil. 2011.

[40] T.L. Saaty. "Decision making with the Analytic Hierarchy Process". International. Journal of Services Sciences. Vol. 1 N $^{\circ}$ 1, pp. 83-98. 2008.

[41] J. Wallenius, J.S. Dyer, P.C. Fishburn, R.E. Steuer, S. Zionts and K. Deb. "Multiple criteria decision making, multiple attribute utility theory". Management Science. Vol. 54 $\mathrm{N}^{\circ}$ 7, pp. 1336-1349. 2008.

[42] M. Doumpos and C. Zopounidis. "Multiplecriteria decision aid classification methods". Kluwer. Dordrecht, Holanda. 2002.

[43] G.H. Tzeng and J.J. Huang. "Multiple attribute decision making: methods and applications". Chapman and Hall/CRC. Boca Raton, Estados Unidos. 2011.

[44] A. Ishizaka and P. Nemery. "Multi-criteria decision analysis: methods and software". John Wiley \& Sons Ltd. Chichester, Estados Unidos. 2013.
[45] J.W. Creswell. "Research design: qualitative, quantitative, and mixed methods approaches". Sage Publications. Thousand Oaks, Estados Unidos. 2002.

[46] O. Ustun and E.A. Demirtas. "Multi-period lot-sizing with supplier selection using achievement scalarizing functions". Computers and Industrial Engineering. Vol. 54, pp. 918931. 2008.

[47] V.N. Sundharam, V. Sharma and I.S. Stephan Thangaiah. "An integration of BSC and AHP for sustainable growth of manufacturing industries". International Journal of Business Excellence. Vol. 6 No 1, pp. 77-92. 2013.

[48] E. Keshavarz, M. Ftahikenari, A. Rohani and S.M. Bagheri. "Performance evaluation of banks using balanced scorecard". International Journal of Business Excellence. Vol. $7 \mathrm{~N}^{\circ} 3$, pp. 371-393. 2014.

[49] L.F.A.M. Gomes, M.C.G. Araya e C. Carignano. "Tomada de decisões em cenários complexos: introdução aos métodos discretos de apoio multicritério à decisão. Cengage Learning. São Paulo, Brasil. 2004.

[50] S. Greco, M. Ehrgott and J.F. Figueira. "Trends in multiple criteria decision analysis". Springer. New York, Estados Unidos. 2012.

[51] T.L. Saaty and L.G. Vargas. "Methods, concepts \& applications of the Hierarchy Process". Springer. New York, Estados Unidos. 2012.

[52] F.A.S. Marins, M.S. Pereira, M.C.N. Balderrain e L.M.S. Urbina. "Métodos de tomada de decisão com múltiplos critérios: aplicações na indústria aeroespacial". Blucher Acadêmico. São Paulo, Brasil. 2010.

[53] T.L. Saaty and J.S. Shang. "An innovative orders-of-magnitude approach to AHP-based multi-criteria decision making: prioritizing divergent intangible humane acts". European Journal of Operational Research. Vol. 214 $\mathrm{N}^{\circ}$ 3, pp. 703-715. 2011.

[54] H.G. Costa. "Auxílio multicritério à decisão: método AHP”. ABEPRO. Rio de Janeiro, Brasil. 2006.

[55] A. Guitouni and J.M. Martel. "Tentative guidelines to help choosing an appropriate MCDAmethod". European Journal of Operational Research. Vol. $109 \mathrm{~N}^{\circ}$ 2, pp. 501521. 1998. 